\title{
Reactive Power and Soft-Switching Capability Analysis of Dual-Active-Bridge DC-DC Converters with Dual-Phase-Shift Control
}

\author{
Huiqing $\mathrm{Wen}^{\dagger}$ and $\mathrm{Bin} \mathrm{Su}^{*}$ \\ 'Department of Electrical and Electronic Engineering, Xi' an Jiaotong-Liverpool University, Suzhou, China \\ ${ }^{*}$ Hangzhou Electric Power Bureau, State Grid Corporation of China, Hangzhou, China
}

\begin{abstract}
This paper focuses on a systematical and in-depth analysis of the reactive power and soft-switching regions of Dual Active Bridge (DAB) converters with dual-phase-shift (DPS) control to achieve high efficiency in a wide operating range. The key features of the DPS operating modes are characterized and verified by analytical calculation and experimental tests. The mathematical expressions of the reactive power are derived and the reductions of the reactive power are illustrated with respect to a wide range of output power and voltage conversion ratios. The ZVS soft-switching boundary of the DPS is presented and one more leg with ZVS capability is achieved compared with the CPS control. With the selection of the optimal operating mode, the optimal phase-shift pair is determined by performance indices, which include the minimum peak or rms inductor current. All of the theoretical analysis and optimizations are verified by experimental tests. The experimental results with the DPS demonstrate the efficiency improvement for different load conditions and voltage conversion ratios.
\end{abstract}

Key words: Bidirectional dc-dc converter, Dual active bridge, Modulation strategy, Reactive power, Soft switching

\section{NOMENCLATURE}

$V_{T 1} \quad$ magnitude of the transformer primary voltage

$v_{T 1} \quad$ transient value of the transformer primary voltage

$V_{T 2} \quad$ magnitude of the transformer secondary voltage

$v_{T 2} \quad$ transient value of the transformer secondary voltage

$I_{S I} \quad$ rms current produced by $V_{S I}$

$I_{L_{-} r m s} \quad$ rms current flowing through the inductor $L$

$V_{L_{-} r m s} \quad$ rms voltage across the inductor $L$

$Q_{L} \quad$ reactive power produced by the inductor $L$

$I_{\text {peak }} \quad$ peak current of the inductor $L$

$I_{\text {peak } \_ \text {min }} \quad$ minimum value of $I_{\text {peak }}$

$I_{r m s_{-} \text {min }} \quad$ minimum value of $I_{L_{-} r m s}$

$I_{\text {peak_min_G }}$ global minimum value of $I_{\text {peak }}$

$I_{\text {peak_min_L }} \quad$ local minimum value of $I_{\text {peak }}$

Manuscript received May 25, 2014; accepted Aug. 12, 2014

Recommended for publication by Associate Editor Bor-Ren Lin.

${ }^{\dagger}$ Corresponding Author: huiqing.wen@xjtlu.edu.cn

Tel: + 86-0512-81880477, Xi'an Jiaotong-Liverpool University

*Hangzhou Electric Power Bureau, State Grid Corporation of China, China

\section{INTRODUCTION}

Originally proposed in [1] for aerospace power applications, the topology of a dual active bridge (DAB) converter became popular in battery chargers [2], hybrid wind-photovoltaic systems [3], solid-state transformers (SSTs) [4], micro grids [5], and hybrid electric vehicles [6]. Compared with other isolated dc-dc topologies, the DAB converter shows many advantages, such as bi-directional power flow, inherent soft-switching capability, power controllability and high efficiency [7].

A DAB DC-DC converter typically consists of two full bridges $\left(B_{1}\right.$ and $\left.B_{2}\right)$ that are interconnected through a high frequency transformer $T_{r}$, as shown in Fig. 1. The two bridges are composed of the switches of $Q_{11}-Q_{14}$ and $Q_{21}-Q_{24}$, respectively. The modulation usually adopted is the conventional phase-shift (CPS) technique. The main advantage of the CPS lies in its implementation simplicity because the averaged active power can be directly regulated by the phase shift angle. However, the CPS has the drawbacks of high reactive power and circulating current flowing through the transformer, especially when the voltage of $N V_{T 1}$ is different from $V_{T 2}$, which leads to high conduction 
losses [1]. The DAB converter may also lose the soft switching capability when the voltage conversion ratio deviates from unity [8]. However, it is difficult to always fulfill a voltage ratio of $1: 1$ in practical applications considering the wide operating voltage range of energy storage sources such as batteries or ultracapacitors [9]. As a result, the CPS suffers from a low power conversion efficiency due to its high reactive power and hard switching operation [10], [11].

In order to improve the efficiency of the DAB over a wide operating voltage range, various control algorithms have been proposed. These include a phase-shift plus pulse width modulation (PSPWM) control strategy [8], [11]-[12], triangular modulation (TRM) [13], trapezoidal modulation (TZM) [14] and hybrid modulation [15]. In addition, the pulse-skipping control strategy is also proposed for grid-tied converter applications to improve the efficiency for light loads [16]. However, the pulse-skipping control strategy shows a negative impact on the normal operation of systems [16]. The PSPWM is ruled by two manipulated variables, which include the modulation index and the phase shift between the transformer primary and secondary voltages. With PSPWM, the bridge of the DAB converter with duty-cycle control varies with the voltage conversion ratio and power flow direction, which increases the implementation complexity [7]. Furthermore, the proposed PSPWM with negative values of phase shift shows only half of the attainable maximum output power compared with that of the CPS [8]. The TRM and TZM focus on minimizing the current of the switching devices at the turn-off instant to reduce the switching loss. However, they might increase the rms current stress on the transformer and can only be adopted for a limited operating range. Hybrid modulation switches the modulation strategies among the TRM, TZM and CPS with respect to different operation stages. The hybrid modulation has a complicated implementation because various strategies are involved and the operating status of the converter needs to be monitored in real time.

A dual-phase-shift (DPS) control is proposed to eliminate the reactive power and then minimize the conduction losses of the DAB converters for electric vehicle applications [17]. In [7], various control strategies are compared in terms of maximum output power, efficiency, ZVS range and practical control. The conclusion of this reference is that the "DPS control may be relative optimal method for the large-scale practical application from the implementation difficulty and performance." However, an experimental comparison is conducted in [18] to evaluate the performance of both the CPS and the DPS. It shows that the efficiency improvement using the DPS is not as good as expected. The effect of the DPS in in terms of efficiency improvement is also undervalued in other studies [6], [8], [19], [20]. The possible reasons may include:
1) The operating modes of the DPS: there are four operating modes with respect to the characterization of the phase-shift pairs. The power characteristics of these operating modes are difference. The optimal operating modes must be determined while considering the voltage conversion ratio and load conditions.

2) Soft-switching range: the performance characteristic of the DAB converter, especially the soft-switching range with the DPS, should be presented while considering applications with variable loads.

3) The quantitive comparison of the reactive power reduction with the DPS: considering the complexity of the DPS, the analysis of the reactive power in [17] only focuses on one special operation condition. However, more general expressions should be given for various operating conditions and the illustration of the reactive power reduction with different operating modes should be provided quantitatively.

4) The complexity for implementation: the study in [6] demonstrates that the 2-D solving problem of the DPS, which is regarded as too complex to be optimized when compared with 1-D PWM strategies. However, like PSPWM [10], the efficiency optimization with the DPS is essentially a 1-D problem.

In this paper, the reactive power and zero voltage switching (ZVS) soft-switching capability of DAB converters with DPS are analyzed. Section II presents a power flow analysis of the DPS and its four operating modes. In section III, the features of the four operating modes are defined to determine the optimal one while considering practical operating conditions. Section IV shows the experimental results, which reveal that the DPS can expand the ZVS operating range and enhance the overall efficiency.

\section{POWER FLOW ANALYSIS WITH DPS}

DPS manipulates two phase shifts, where $D_{l}$ symbolizes the phase shift between the diagonal control signals in the same bridge, for instance, between the gate signals of $Q_{11}$ and $Q_{14}$, and $D_{2}$ is the phase shift between the primary and the corresponding secondary gate signals, for instance, between the gate signals of $Q_{11}$ and $Q_{21}$. All of the switching devices using DPS are operated at a 50\% duty-cycle, which is consistent with the CPS. Various operating conditions exist in DPS with respect to the power flow direction and buck/boost operation. In the forward mode, the transformer primary voltage $v_{T 1}$ leads the transformer secondary voltage $v_{T 2}$ so that power flows from $V_{S I}$ to the load. When $v_{T I}$ lags $v_{T 2}$, the DAB operates in the backward mode, where the power flow is transferred from right to left. The voltage conversion ratio $d$ is defined as:

$$
d=V_{T 2} /\left(N V_{T 1}\right)
$$

When the DPS operates in the boost condition $(d>1)$, the main waveforms with four operating modes are shown in Fig. 


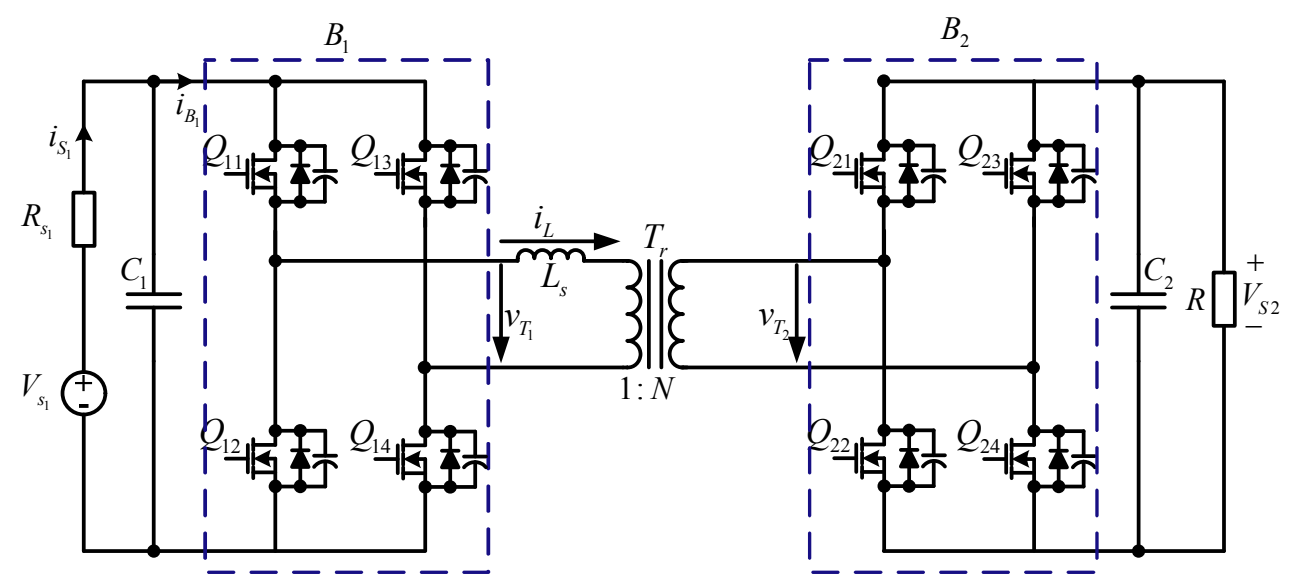

Fig. 1. Schematics of dual-active bridge converters.

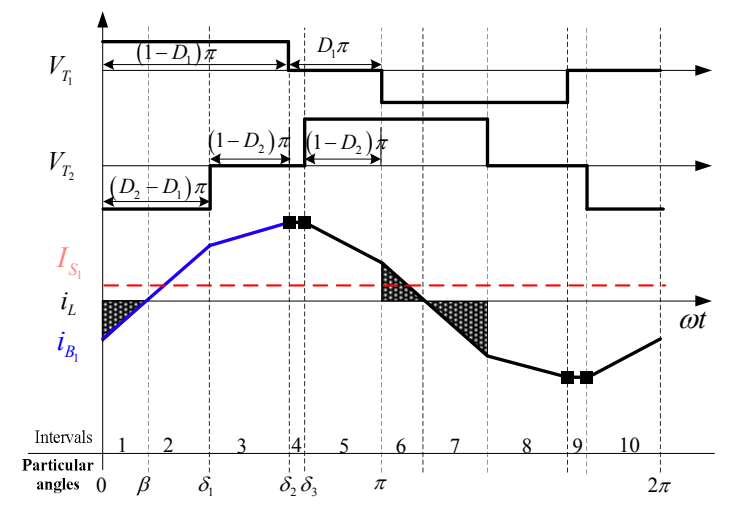

(a)

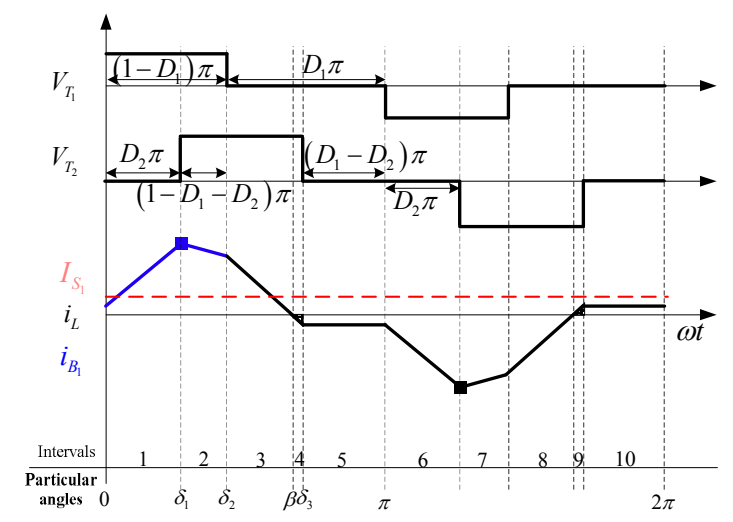

(c)

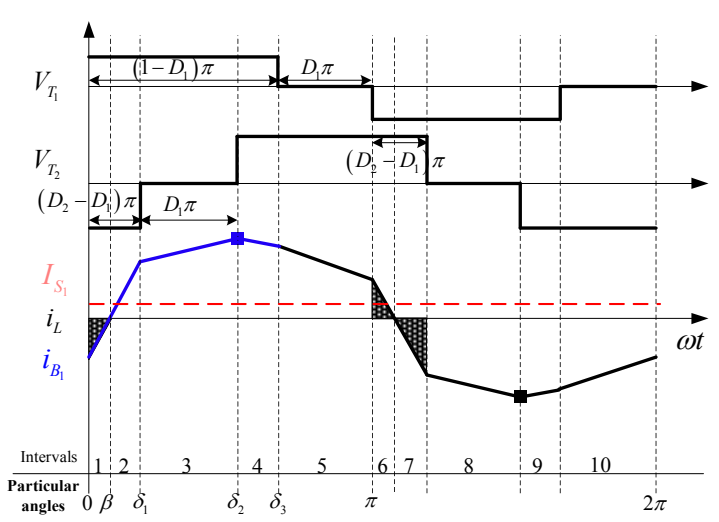

(b)

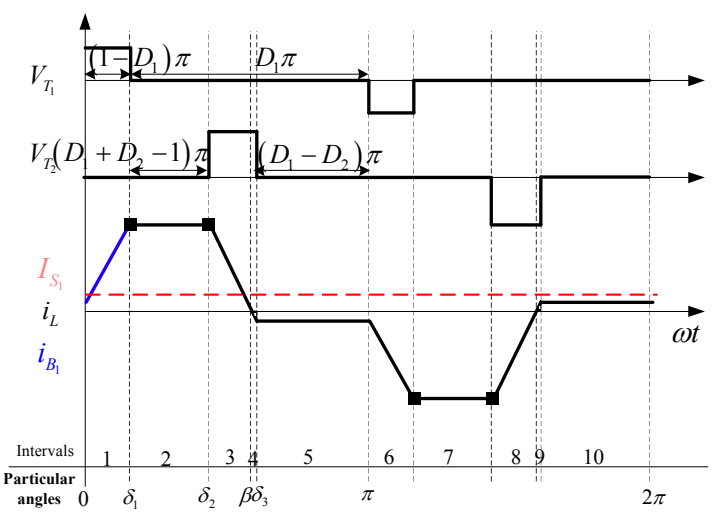

(d)

Fig. 2. Operating modes and typical waveforms of the DAB converter with DPS in the boost operation condition. (a) DPS_I: $\left(D_{2}>D_{1}\right) \wedge\left(D_{2}+D_{1} \geq 1\right)$. (b) DPS_II: $\left(D_{2}>D_{1}\right) \wedge\left(D_{2}+D_{1}<1\right)$. (c) DPS_III: $\left(D_{2} \leq D_{1}\right) \wedge\left(D_{2}+D_{1}<1\right)$. (d) DPS_IV: $\left(D_{2} \leq D_{1}\right) \wedge\left(D_{2}+D_{1} \geq 1\right)$.

2, where $\beta$ corresponds to the zero crossing instant of the inductor current $i_{L}$, and $\delta_{1} \sim \delta_{3}$ represent the switching angles. The meaning of $v_{T 1}, v_{T 2}$ and $I_{S 1}$ are defined in Fig. 1, and their definitions are also explained in the nomenclature. The blue line $i_{B 1}$ indicates the transient current to bridge $B_{l}$, which corresponds to the positive part of the transformer primary voltage $v_{T 1}$. The red line $I_{S l}$ represents the current produced by voltage source $V_{S I}$. Considering that the variation of the input voltage is small, the current in capacitor $C_{l}$ can be neglected. Thus, the relationship between $i_{B I}$ and $I_{S I}$ can be expressed as:

$$
\frac{1}{2 \pi} \int_{0}^{2 \pi} i_{B 1} d \omega t=I_{S 1}
$$

To analyze the optimal operating mode, $I_{S I}$ is kept constant 
TABLE I

EXPRESSIONS OF CURRENT AT THE SWITCHING ANGLES WITH DPS (PU)

\begin{tabular}{c|c|c|c|c|c}
\hline \hline Modes & $i_{L}(0)$ & $i_{L}\left(\delta_{1}\right)$ & $i_{L}\left(\delta_{2}\right)$ & $i_{L}\left(\delta_{3}\right)$ & $i_{L}(\pi)$ \\
\hline I & $D_{1}+d+d D_{1}-2 d D_{2}-1$ & $-D_{1}+d-d D_{1}+2 D_{2}-1$ & $\left(1-D_{1}\right)(d+1)$ & $\left(1-D_{1}\right)(d+1)$ & $-D_{1}-d-d D_{1}+2 d D_{2}+1$ \\
\hline II & $D_{1}+d+d D_{1}-2 d D_{2}-1$ & $-D_{1}+d-d D_{1}+2 D_{2}-1$ & $D_{1}+d-d D_{1}+2 D_{2}-1$ & $-D_{1}-d+d D_{1}+2 d D_{2}+1$ & $-D_{1}-d-d D_{1}+2 d D_{2}+1$ \\
\hline III & $\left(1-D_{1}\right)(d-1)$ & $D_{1}+d-d D_{1}+2 D_{2}-1$ & $-D_{1}-d+d D_{1}+2 d D_{2}+1$ & $\left(D_{1}-1\right)(d-1)$ & $\left(D_{1}-1\right)(d-1)$ \\
\hline IV & $\left(1-D_{1}\right)(d-1)$ & $\left(1-D_{1}\right)(d+1)$ & $\left(1-D_{1}\right)(d+1)$ & $\left(D_{1}-1\right)(d-1)$ & $\left(D_{1}-1\right)(d-1)$ \\
\hline \hline
\end{tabular}

TABLE II

Expressions of Average Output Power, Inductor RMS Current And Peak Current (Pu)

\begin{tabular}{|c|c|c|c|}
\hline Modes & $P_{\text {out }}$ & $I_{L_{-} r m s}$ & $I_{\text {peak }}$ \\
\hline I & $d\left(1-D_{2}\right)\left(1+D_{2}-2 D_{1}\right)$ & $\begin{array}{l}\sqrt{2 D_{1}^{3} d^{2}+4 D_{1}^{3} d+2 D_{1}^{3}-3 D_{1}^{2} d^{2}-6 D_{1}^{2} d-3 D_{1}^{2}+12 D_{1} D_{2}^{2} d} \\
-24 D_{1} D_{2} d+12 D_{1} d-4 D_{2}^{3} d+12 D_{2} d+d^{2}-6 d+1\end{array}$ & $\left(1-D_{1}\right)(d+1)$ \\
\hline II & $d\left(-D_{1}^{2}-2 D_{2}^{2}+2 D_{2}\right)$ & 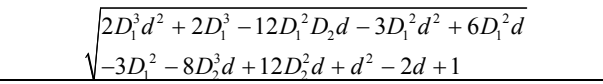 & $D_{2}(1+d)+\left(1-D_{1}-D_{2}\right)|1-d|$ \\
\hline III & $d\left(2-2 D_{1}-D_{2}\right) D_{2}$ & $\sqrt{\begin{array}{l}2 D_{1}^{3} d^{2}-4 D_{1}^{3} d+2 D_{1}^{3}-3 D_{1}^{2} d^{2}+6 D_{1}^{2} d-3 D_{1}^{2} \\
-12 D_{1} D_{2}^{2} d-4 D_{2}^{3} d+12 D^{2} d+d^{2}-2 d+1\end{array}}$ & $D_{2}(1+d)+\left(1-D_{1}-D_{2}\right)|1-d|$ \\
\hline IV & $d\left(1-D_{1}\right)^{2}$ & $\sqrt{\left(D_{1}-1\right)^{2}\left(2 D_{1}-6 d+12 D_{2} d+2 D_{1} d^{2}+d^{2}+1\right)}$ & $\left(1-D_{1}\right)(d+1)$ \\
\hline
\end{tabular}

TABLE III

Comparison of the Operating Modes with Regard to the Defined Features

\begin{tabular}{c|c|c|c}
\hline \hline Features & DPS_I & DPS_II & DPS_III \\
\hline The ratio of reactive current & Large & Large & Small \\
\hline The overlap of $v_{T 1}$ and $v_{T 2}$ & No & Yes & Yes \\
\hline Circulating current & Peak Current & No & Small Current \\
\hline The duration of $v_{T 1}$ and $v_{T 2}$ & Possible long & Possible long & Possible long \\
\hline \hline
\end{tabular}

in Fig. 2, which represents the same output power. The reactive power of each operating mode, which is represented by the dark shaded area in Fig. 2, for both the input and output sides, is examined and compared. The peak current is marked with a square to symbolize the power transfer features of each mode. With the dynamics of $i_{L}$, shown in Fig. 2 , the analytical expressions of the current $i_{L}$ at the switching angles $\delta_{1}, \delta_{2}, \delta_{3}$ and $\pi$ for each operating mode of the DPS can be derived and are shown in Table I. Due to the boost operation, the inductor current at the initial condition, $i_{L}(0)$, is larger than zero for DPS_III and DPS_IV. The average output power $P_{o}$, the inductor rms current $I_{L r m s}$, and the inductor peak current $I_{p e a k}$, for the four operating modes are derived accordingly and shown in Table II. All of the quantities shown in Table I and Table II are normalized by the following base values:

$$
I_{b}=V_{S 1} /\left(4 L_{s} f_{s}\right), P_{b}=V_{S 1}^{2} /\left(4 L_{s} f_{s}\right)
$$

where, $f_{s}$ is the switching frequency, and the variables $V_{S 1}, f_{s}$ and $L_{s}$ only affect the magnitude of $P_{o}$. The phase-shift pair of $D_{1}$ and $D_{2}$ solely determines the operating modes and the amount of output power.

\section{IMPLEMENTATION OF THE DPS CONTROL}

\section{A. Optimal Operating Mode}

The optimal operating mode of the DPS should be determined with regard to the voltage conversion ratio and the output power range. Four distinct operating modes exist and their main features must be grasped. In this section, these major features are defined and summarized as follows.

1) The Ratio of the Reactive Current: The percentage of reactive current at both the output and input sides are considered. For DPS_III and DPS_IV in the boost operation, the initial inductor current meets $i_{L}(0) \geq 0$ according to Table I. Thus, the reactive power at the input side is zero for the boost operation because the phase of the inductor current $i_{L}$ is always the same as the transformer primary voltage $v_{T l}$, as shown in Fig. 2(c) and Fig. 2(d). Without the reactive current, the conducting current is largely reduced.

2) The Overlap of $v_{T 1}$ and $v_{T 2}$ : With the DPS, both the transformer primary voltage $v_{T 1}$ and the secondary voltage $v_{T 2}$ show three levels, including positive, zero, and negative. The period that $v_{T 1}$ and $v_{T 2}$ have the same polarity such as positive, is defined as the overlap of $v_{T 1}$ and $v_{T 2}$. It is desirable to constrain the change rate of the inductor current, $i_{L}$, and to reduce the current peak due to voltage cancellation.

3) The Circulating Current: The circulating current exists when both $v_{T 1}$ and $v_{T 2}$ are zero-level. Two devices with the same position in a bridge (upside or downside) are conducting for this period. There is no energy transferred between the input, the output and the leakage inductance in 
TABLE IV

SOFT-SWITCHING RESTRICTIONS AND BOUNDARIES WITH DPS

\begin{tabular}{c|c|c|c|c|c|c|c|c}
\hline \hline Mode & \multicolumn{5}{|c|}{ Constraints } & \multicolumn{3}{c}{ Boundaries } \\
\hline I & $i_{L}(0)<0$ & $i_{L}\left(\delta_{3}\right)>0$ & $i_{L}\left(\delta_{2}\right)>0$ & $i_{L}\left(\delta_{1}\right)>0$ & $D_{2}>\frac{D_{1}(d+1)+(d-1)}{2 d}$ & \multicolumn{2}{c}{ Always } & Always \\
\hline II & $i_{L}(0)<0$ & $i_{L}\left(\delta_{2}\right)>0$ & $i_{L}\left(\delta_{3}\right)>0$ & $i_{L}\left(\delta_{1}\right)>0$ & $D_{2}>\frac{D_{1}(d+1)+(d-1)}{2 d}$ & $D_{2}>\frac{\left(1-D_{1}\right)(1-d)}{2}$ & $D_{2}>\frac{D_{1}(d+1)+(1-d)}{2}$ \\
\hline III & $i_{L}(0)<0$ & $i_{L}\left(\delta_{1}\right)>0$ & $i_{L}\left(\delta_{2}\right)>0$ & $i_{L}\left(\delta_{3}\right)<0$ & Always for Buck & $D_{2}>\frac{\left(1-D_{1}\right)(1-d)}{2}$ & $\left.D_{2}\right)$ \\
\hline IV & $i_{L}(0)<0$ & $i_{L}\left(\delta_{2}\right)>0$ & $i_{L}\left(\delta_{1}\right)>0$ & $i_{L}\left(\delta_{3}\right)<0$ & Always for Buck & Always & $D_{1}(d+1)+(1-d)$ \\
2 & Always for Boost \\
\hline \hline
\end{tabular}

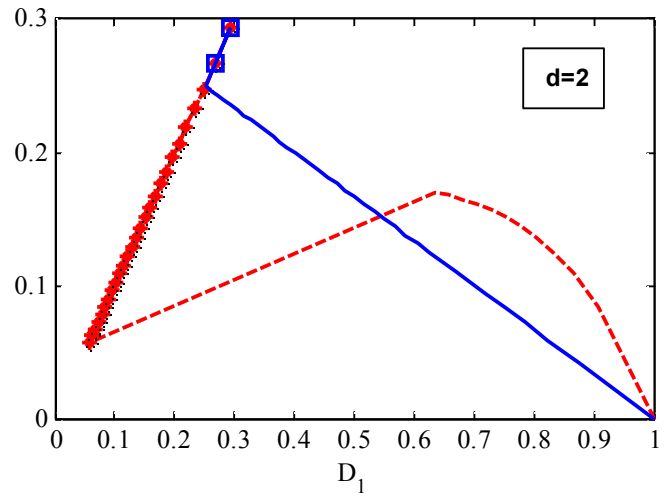

Fig. 3. The optimal trajectory of the phase-shift pair for the minimum peak current $\left(I_{\text {peak min }}\right)$ and the minimum rms current $\left(I_{r m s \text { min }}\right)$ with DPS_III and the meaning of different types of lines and marks representing $I_{\text {peak min } G}$ (solid blue line), $I_{\text {peak min } L}$ (solid blue line with square marks), $I_{r m s} \min G$ (dashed red line) and $I_{r m s_{-} \min _{-} L}$ (dashed red line with star marks) respectively.

the circulating period. However, this increases the rms current without any change in the delivered power. In particular, for DPS_I and DPS_IV, the rms current will be significantly increased because the circulating current is the inductor peak current.

4) The Duration of $v_{T 1}$ and $v_{T 2}$ : With the DPS, the duration of $v_{T 1}$ and $v_{T 2}$ with positive voltages are same for all of the operating modes and expressed as $\left(1-D_{I}\right)$. However, the range is different for each operating mode according to the corresponding combination of phase-shift pairs. For DPS_IV, the duration of $v_{T 1}$ and $v_{T 2}$ is the shortest. Thus, both its rms and peak current are high.

Table III illustrates a comparison of the four operating modes with the DPS in terms of the defined features. Both DPS_II and DPS_III are good candidates for the optimal operating mode according to this trade-off. However, considering that the circulating current can be tuned to zero by the selection of the phase-shift pairs for DPS_III and it is difficult to reduce the reactive power a lot with DPS_II, DPS_III is selected as the optimal operating mode. This trade-off result, which is based on the aforementioned features, will be verified by analytical calculations and experimental tests in the following sections.

\section{B. Determination of the Phase-Shift Pairs}

With the selection of the optimal operating mode while considering the output power range, the optimal phase-shift pair should be determined based on performance indices. In this paper, the inductor rms current $I_{L_{r} r m s}$ and the inductor peak current $I_{\text {peak }}$ are chosen as the performance indices because $I_{\text {peak }}$ is linked with the switching losses and $I_{L_{-} r m s}$ largely determines the conducting losses and transformer losses. The mathematical expressions for the four operating modes of the DPS are shown in Table II. The constraint condition in the optimization process is the average output power $P_{o}$. The expressions shown in Table II indicate that the performance indices vary with the phase-shift pair and voltage ratio. Considering that the voltage ratio is a given parameter and not a controllable variable, only the phase-shift pairs can be tuned to optimize these performance indices with the constraint of the output power requirement.

Taking the operation of the DPS_III as example to illustrate the process of determining the optimal phase-shift pairs, the inner phase-shift variable $D_{l}$ can be expressed as (4), which is a function of the transferred power flow.

$$
D_{1}=1-\frac{D_{2}}{2}-\frac{2 P_{o} f_{s} L_{s}}{d D_{2} V_{s_{1}}^{2}}
$$

The output power with the DPS is symmetrical with $D_{2}=0.5$, and the performance indices of both the peak current $I_{\text {peak }}$ and the rms current $I_{L_{-} r m s}$ are lower in the range of $D_{2} \leq 0.5$ when compared with the other range. Thus, the range of the outer phase-shift variable is set to vary between 0 and 0.5 .

The trajectory of the phase-shift pair can be obtained to achieve either the minimum peak current $I_{\text {peak min }}$ or the minimum rms current $I_{r m s \text { min }}$. Two kinds of minimum points exist in the optimization process: one is the global minimum point for the whole range and the other is the local minimum point with respect to the range of the phase-shift pairs for each operating mode. Taking the minimum peak current point as an example, with the optimal point of the phase-shift pair, the output power plane and the peak current plane should be met at the global minimum peak current $I_{\text {peak_min_G }}$. Thus, the gradients of the output power and the performance index of the inductor peak current should be parallel in $D_{1}$ and $D_{2}$ coordination. The corresponding expression is shown as:

$$
\left(\frac{\partial I_{\text {peak }}}{\partial D_{1}}, \frac{\partial I_{\text {peak }}}{\partial D_{2}}\right)=\left(\frac{\partial P_{\text {out }}}{\partial D_{1}}, \frac{\partial P_{\text {out }}}{\partial D_{2}}\right)
$$

The expression for the optimal phase-shift pair to achieve the global minimum peak current $\left(D_{I_{-} \text {Ipeak_min_G }}, D_{2 \text { _Ipeak_min_G }}\right)$ 


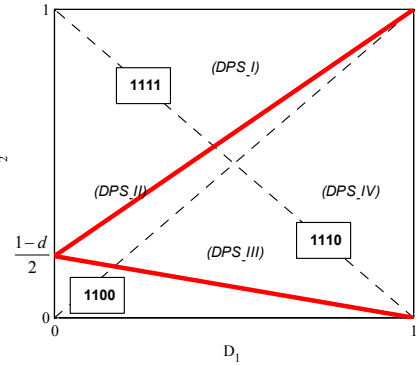

(a) $d<1$

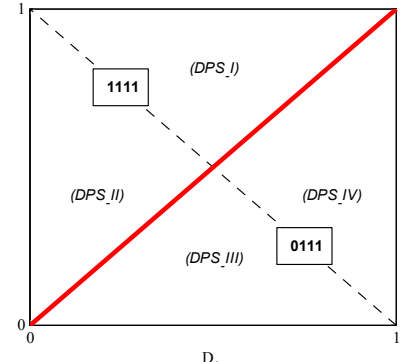

(b) $d=1$

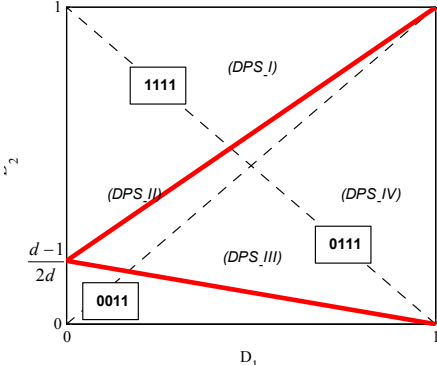

(c) $d>1$

Fig. 4. ZVS soft-switching boundaries for four phase legs with respect to the operating modes of DPS and voltage conversion ratios.

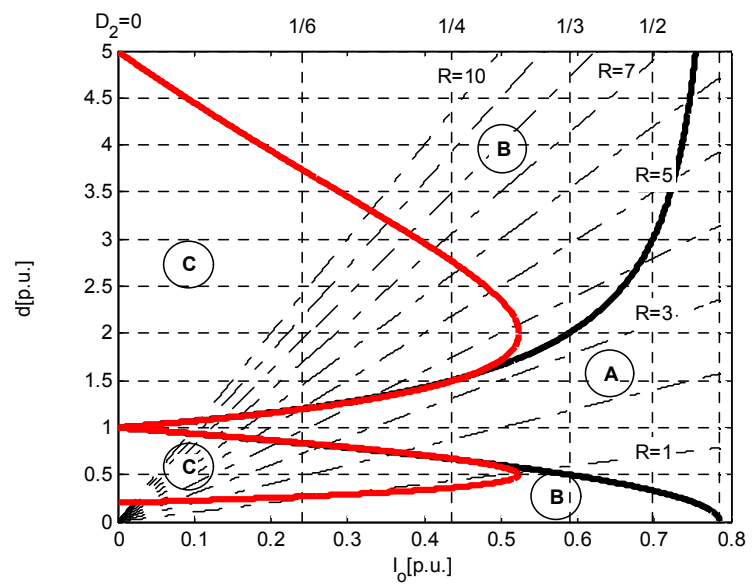

Fig. 5. Soft-switching regions of DAB converter as a function of $d$ and $I_{o}$ [p.u.] with $R$ as parameter.

A) -Soft-switching region using CPS;

B) - Soft-switching region using the DPS;

C) - Hard-switching region using the DPS.

can be obtained as:

$$
\begin{aligned}
& \int D_{1_{-} I_{\text {peek } \_ \text {min_G }}}=2-\frac{2 D_{2_{-} I_{\text {peak } \_ \text {min_G } G}}}{1-d} \\
& \left\{D_{2_{-} I_{\text {pack } \_ \text {min_G }}}=\frac{(1-d) V_{s_{1}}+\sqrt{(1-d)\left(-V_{s_{1}}^{2} d^{2}+V_{s_{1}}^{2} d+4 L_{s} f_{s} d P_{o}+12 L_{s} f_{s} P_{o}\right) / d}}{(d+3) V_{s_{1}}}\right.
\end{aligned}
$$

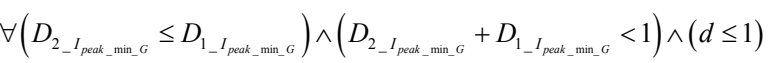

$$
\begin{aligned}
& \int D_{1_{-} I_{\text {peak } \_ \text {min_G } G}}=2-\frac{2 d D_{2_{-} I_{\text {peak } \_ \text {min } G} G}}{d-1} \\
& \left\{D_{2_{-} I_{\text {peat_nin_G }}}=\frac{(d-1) V_{s_{1}}+\sqrt{(d-1)\left(V_{s_{1}}^{2} d^{2}-V_{s_{1}}^{2} d+12 L_{s} f_{s} d P_{o}+4 L_{s} f_{s} P_{o}\right) / d}}{(3 d+1) V_{s_{1}}}\right.
\end{aligned}
$$

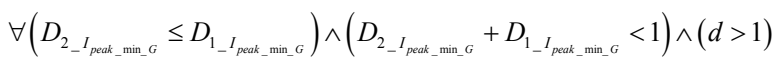

As shown in this expression, the determination of the optimal phase-shift pair with the DPS is a 1-D optimization problem, which is similar to the PSPWM shown in [10].

The local minimum peak current $I_{\text {peak min } L}$ can also be determined by the following numerical equation:

$$
I_{\text {peak } \_ \text {min_L } L}=\min \left(I_{\text {peak }}\left(V_{\text {in }}, d, f_{s}, L, P_{o}, D_{1}, D_{2}\right)\right) \forall\left(D_{2} \leq D_{1}\right) \wedge\left(D_{2}+D_{1}<1\right)
$$

Similarly, the minimum rms current $I_{\text {rms_min }}$ can be determined by the analytical calculation and numerical
TABLE V

Reactive Power for DifFerent Operating Modes of DPS

\begin{tabular}{c|c}
\hline \hline Modes & $Q_{L}$ \\
\hline I & $\sqrt{\left[\left(1-D_{1}\right) d^{2}+\left(2 D_{2}-2 D_{1}\right) d+1-D_{1}\right]\left(2 D_{1}^{3} d^{2}+4 D_{1}^{3} d+2 D_{1}^{3}-3 D_{1}^{2} d^{2}-6 D_{1}^{2} d\right.}$ \\
& $\sqrt{\left.-3 D_{1}^{2}+12 D_{1} D_{2}^{2} d-24 D_{1} D_{2} d+12 D_{1} d-4 D_{2}^{3} d+12 D_{2} d+d^{2}-6 d+1\right)}$ \\
\hline II & $\sqrt{\left[\begin{array}{l}\left.\left[1-D_{1}\right) d^{2}+\left(4 D_{2}-2\right) d+1-D_{1}\right]\left(2 D_{1}^{3} d^{2}+2 D_{1}^{3}-12 D_{1}^{2} D_{2} d\right. \\
\left.-3 D_{1}^{2} d^{2}+6 D_{1}^{2} d-3 D_{1}^{2}-8 D_{2}^{3} d+12 D_{2}^{2} d+d^{2}-2 d+1\right)\end{array}\right.}$ \\
\hline III & $\sqrt{\left[\begin{array}{l}\left.\left[1-D_{1}\right) d^{2}+\left(2 D_{1}+2 D_{2}-2\right) d+1-D_{1}\right]\left(2 D_{1}^{3} d^{2}-4 D_{1}^{3} d+2 D_{1}^{3}\right. \\
\left.-3 D_{1}^{2} d^{2}+6 D_{1}^{2} d-3 D_{1}^{2}-12 D_{1} D_{2}^{2} d-4 D_{2}^{3} d+12 D_{2}^{2} d+d^{2}-2 d+1\right)\end{array}\right.}$ \\
\hline IV & $\sqrt{\left[\left(2 D_{1}+1\right)\left(D_{1}-1\right)^{2}+d^{\wedge} 2\left(2 D_{1}+1\right)\left(D_{1}-1\right)^{2}+d\left(12 D_{2}-6\right)\left(D_{1}-1\right)^{\wedge} 2\right]}$ \\
\hline \hline
\end{tabular}

equation (8). The phase-shift trajectory for the minimum rms current is obtained from look-up tables because it is extremely complex to calculate on-line.

$I_{r m s_{-} \min }=\min \left(I_{r m s}\left(V_{i n}, d, f_{s}, L, P_{o}, D_{1}, D_{2}\right)\right) \forall\left(D_{2} \leq D_{1}\right) \wedge\left(D_{2}+D_{1}<1\right)(8)$

Fig. 3 shows the optimal trajectory of the phase-shift pair for the performance indices of the minimum peak current and the minimum rms current with $\mathrm{d}=2$. The red dashed line indicates the phase-shift pairs corresponding to the minimum rms current, and the blue solid line shows the phase-shift pairs corresponding to the minimum peak current. In order to distinguish the global and local optimal phase-shift points, the local optimal phase-shift points $I_{\text {peak min } L}$ and $I_{\text {rms min } L}$ are marked with square and star symbols, respectively. Fig. 3 indicates that the phase-shift trajectories corresponding to the minimum peak current and the minimum rms current are different.

\section{CHARACTERISTIC ANALYSIS WITH DPS}

\section{A. Soft-Switching Range of DPS}

In order to realize ZVS soft-switching operation, the inductor current zero crossing instant should be arranged within the time interval with the voltages $v_{T 1}$ and $v_{T 2}$ having the opposite polarities [8], [10], [21], [22]. Thus, the inductor current $i_{L}$ at different switching angles must fulfill the following inequalities, which are shown in TABLE IV. The resultant boundaries of the soft-switching region for each 


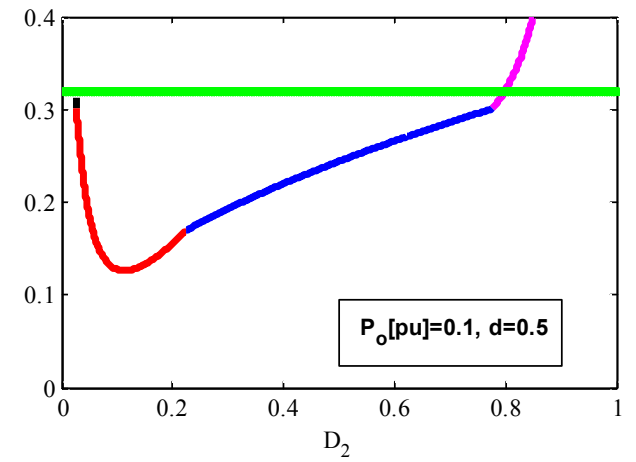

(a)

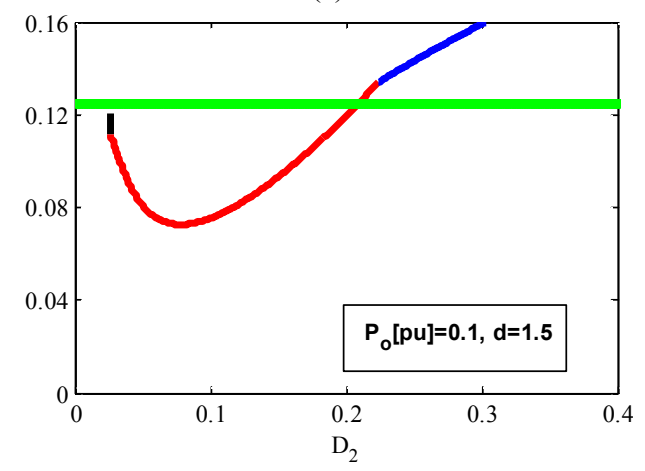

(c)

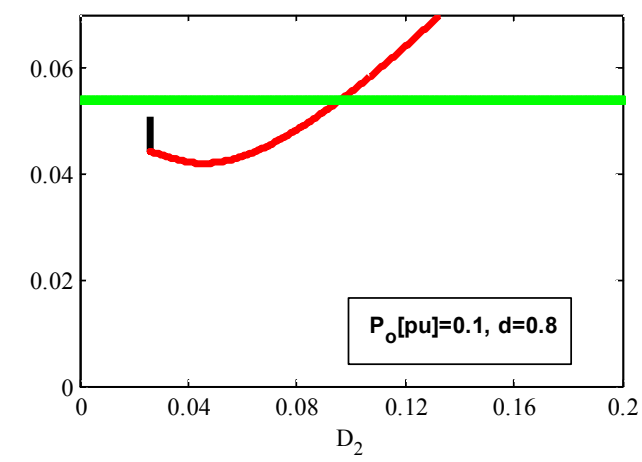

(b)

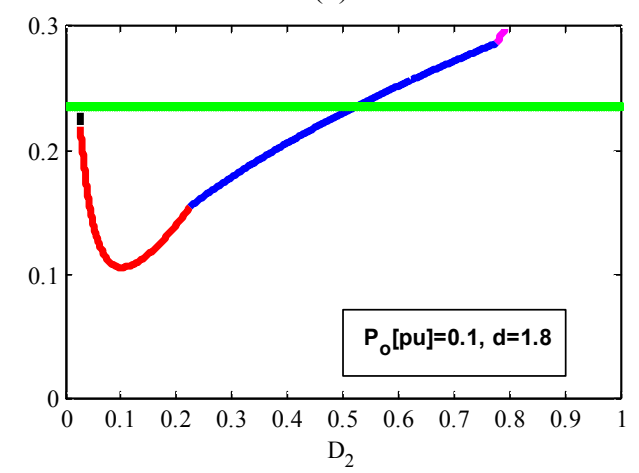

(d)

Fig. 6. Comparison of reactive power with CPS and various operating modes of DPS with regard to the phase-shift angle $D_{2}$ and various voltage conversion ratios $d$ under the condition of low-power $\left(P_{o}[\right.$ p.u.] $=0.1)$.

operating mode are also derived.

Following the derived expressions in Table IV, the ZVS conditions for each phase leg can be illustrated in Fig. 4, which shows the corresponding boundaries of the four phase legs versus the operating mode of the DPS and the voltage conversion ratios. The 4-bit binary numbers represent the availability of ZVS for each phase leg, from left to right, in the DAB topology, which is shown in Fig. 1. When compared with the CPS $\left(D_{I}=0\right)$, the range with ZVS for the four phase legs has been extended by using the DPS. With the two phase-shift variables, $D_{1}$ and $D_{2}$, the DPS is expected to increase the number of phase legs with the ZVS capability. For instance, as shown in Fig. 5 (c), with the CPS, the ZVS condition is " 0011 " when $D_{2}$ is less than the boundary of "(d-1)/(2d)" for the boost operation, while the ZVS conditions can be regulated to " 0111 " by properly selecting the phase-shift pairs using the DPS.

Fig. 5 shows the characteristics of the voltage conversion ratio of the DAB converter versus the output current. The $R$ parameter, shown with the dashdot line, represents the normalized load, and the normalized output current $I_{o}$ [p.u.] can be expressed as (9) for DPS_II.

$$
I_{o}[p . u .]=\frac{P_{o}}{V_{1}^{2} d /\left(\omega L_{s}\right)}=\frac{\left(-\pi D_{1}^{2}-2 \pi D_{2}^{2}+2 \pi D_{2}\right)}{2}
$$

The boundaries of the soft-switching region with the DPS_II control are illustrated in Fig. 5, where the continuous red line represents the boundary for the ZVS soft-switching capability with DPS_II. They are obtained by evaluating (9) with “ $D_{2}=D_{1}=(d-1) /(d+1) \quad$ if $\quad d>1 \quad$ or with “ $D_{2}=D_{1}=(1-d) /(d+1)$ " if $d<1$. The soft-switching region with the CPS control is also illustrated with the continuous black line. The different soft-switching regions in the two-dimensional $d-I_{o}$ plane are marked with "A", "B", and "C". The meaning of these marks are given in the captions of Fig. 5. When compared with one pattern of the PSPWM, which adopts a positive phase-shift angle, the DPS has a wider range when the voltage conversion ratio is far from unity $(d>2)$. However, the DPS cannot extend the region under soft-switching into the whole operation range, as shown in [8], by using another pattern of PSPWM, which adopts a negative phase-shift angle. In addition, a ZVS region at zero loads is also achieved by using the DPS.

\section{B. Reactive Power Minimization}

In this paper, the reactive power is defined as in [8], [23] and TABLE $\mathrm{V}$ shows the reactive power expressions for the different operating modes of the DPS.

The reactive power with the CPS and various operating modes of the DPS with regard to the phase-shift angle $D_{2}$ and various voltage conversion ratios $d$ are illustrated in Fig. 6 for the condition of a low-power $\left(P_{o}[\mathrm{p} . \mathrm{u}]=0.1.\right)$. The reactive power with the CPS is also presented as a benchmark. The different types of lines are defined as follows: CPS (green 


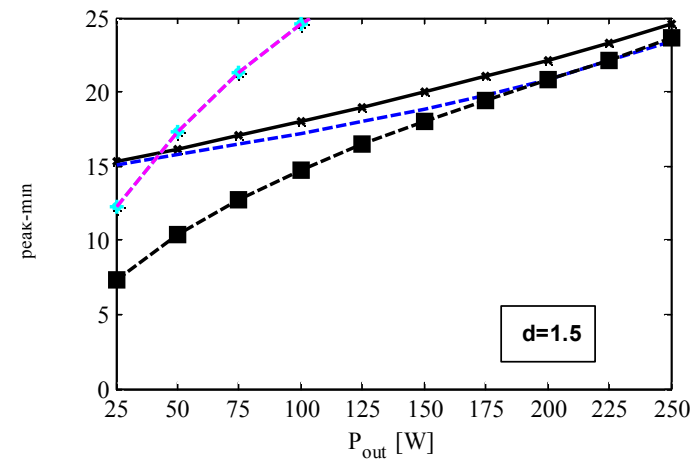

(a)

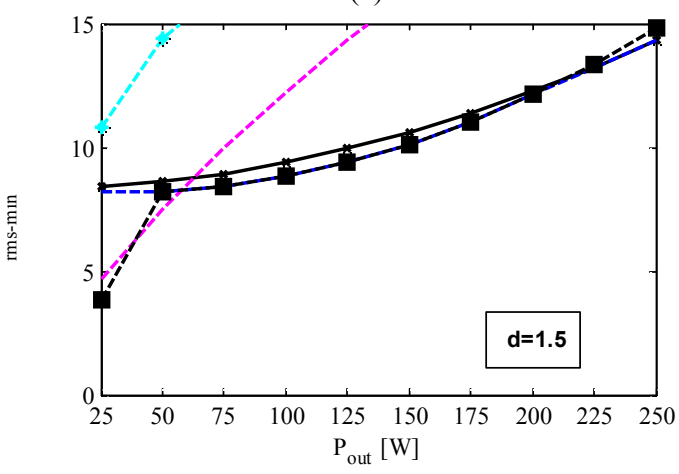

(c)

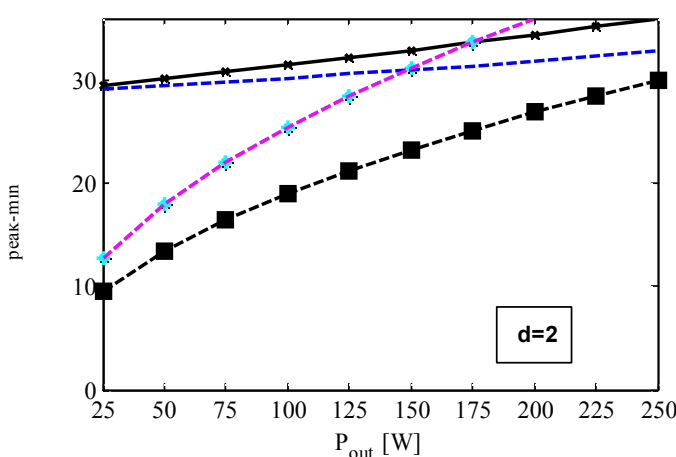

(b)

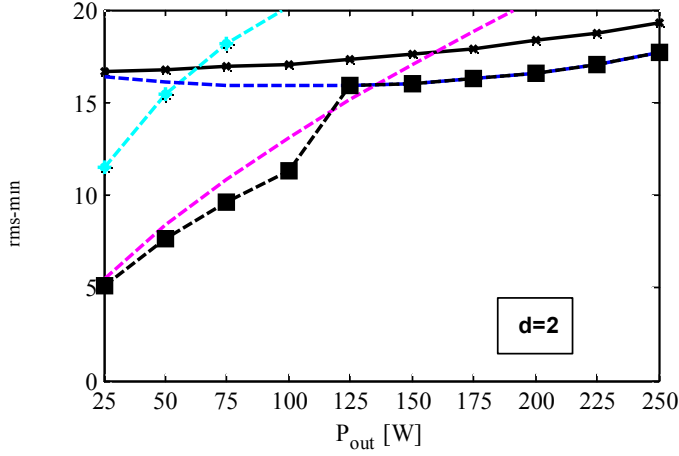

(d)

Fig. 7. Performance comparison of $\mathrm{DAB}$ converter with optimization target of the minimum inductor peak current (top) and the minimum rms current (bottom) considering all operating modes with DPS for the boost operation $(d=1.5,2)$; the meaning of different types of lines and marks representing CPS (solid black line with x-marks), DPS_I (dashed cyan line with star marks), DPS_II (dashed blue line), DPS_III (dashed black line with square marks) and DPS_IV (dashed magenta line) respectively.

TABLE VI

PARAMETERS OF THE DAB CONVERTER PROTOTYPe

\begin{tabular}{rc}
\hline \hline Item & Parameter \\
\hline Inductance $L_{s}(\mu \mathrm{H})$ & 1.73 \\
Transformer turn ratio $N$ & 6 \\
Switching frequency $f_{s}(\mathrm{kHz})$ & 100 \\
Dead time $T_{d}(\mu \mathrm{s})$ & 0.2 \\
Voltage Conversion Ratio $d$ & $0.6 \sim 2$ \\
Maximum output power $P_{\text {omax }}(\mathrm{W})$ & 250 \\
\hline \hline
\end{tabular}

line), DPS I (magenta line), DPS II (black line), DPS III (red line) and $D P S I V$ (blue line).

As shown in Fig. 6, for low-power applications, the DPS can reduce the reactive power in a wide range of voltage conversion ratios. Specifically, all of the operating modes of the DPS can reduce the reactive power in the case of " $d=0.5$ ". Three modes of the DPS show improvement in the case of " $d=1.8$ ", and two modes show improvement in the other cases. The effective range of the outer phase-shift angle $D_{2}$ in reactive power reduction also changes with the voltage conversion ratio $d$. As shown in Fig. 6, the effective ranges of $D_{2}$ in terms of reactive power minimization can be specified as $(0,0.8),(0.02,0.1),(0.02,0.2)$ and $(0.02,0.5)$ corresponding to the following values of $d: 0.5,0.8,1.5$, and 2. The operating modes of both DPS II and DPS III show improvement for all the considered cases. However, when considering the practical range of $D_{2}$ in the control implementation, DPS_III is selected as the optimal operating mode for low-power applications.

\section{Comparison of the Performance Indices}

All four of the operating modes with the DPS are considered in the comparison of the performance indices, which include the inductor peak current and rms current. The phase-shift pairs are determined with the methods discussed above. The main parameters for the comparison are shown as follows: $L_{s}=1.73 \mathrm{uH}, f_{s}=100 \mathrm{kHz}, P_{o}=25 \sim 250 \mathrm{~W}$, and the input voltage $V_{\text {in }}$ is $20 \mathrm{~V}$ for boost operation and $40 \mathrm{~V}$ for buck operation.

Fig. 7 illustrates the comparison results of the performance indices. The optimization target of the minimum inductor peak current and the minimum rms current are considered for all of the operating modes with the DPS. The benchmark is the peak and rms current with the CPS. Based on a comparison of the performance indices, which have been optimized for each operating mode, some conclusions can be made and summarized as follows.

1) DPS with CPS: Theoretically, any performance aspect with the DPS should be better than that with the CPS control because an extra phase shift angle has been provided as 
TABLE VII

EXPERIMENTAL COMPARISON OF DIFFERENT OPERATING MODES

\begin{tabular}{c|c|c|c|c|c|c|c|c|c}
\hline \hline $\begin{array}{c}\text { Operating } \\
\text { Mode }\end{array}$ & $V_{S 1}(\mathrm{~V})$ & $I_{\text {in }}(\mathrm{A})$ & $V_{S 2}(\mathrm{~V})$ & $R_{L}(\Omega)$ & $D_{1}$ & $D_{2}(\mathrm{D})$ & $I_{\text {peak }}(\mathrm{A})$ & $I_{\text {rms }}(\mathrm{A})$ & $\eta(\%)$ \\
\hline CPS & 20 & 3.367 & 215.3 & 933 & 0 & 0.061 & 22.4 & 10.9 & 73.7 \\
\hline DPS_I & 20 & 3.532 & 215.6 & 933 & 0.7076 & 0.7808 & 19.2 & 14.8 & 70.5 \\
\hline DPS_II & 20 & 3.272 & 215.5 & 933 & 0.062 & 0.062 & 20.8 & 10.7 & 76.1 \\
\hline DPS_III & 20 & 2.864 & 216.1 & 933 & 0.6616 & 0.1256 & 13.6 & 7.83 & 87.3 \\
\hline DPS_IV & 20 & 2.959 & 215.1 & 933 & 0.719 & 0.281 & 18 & 9.27 & 83.8 \\
\hline \hline
\end{tabular}

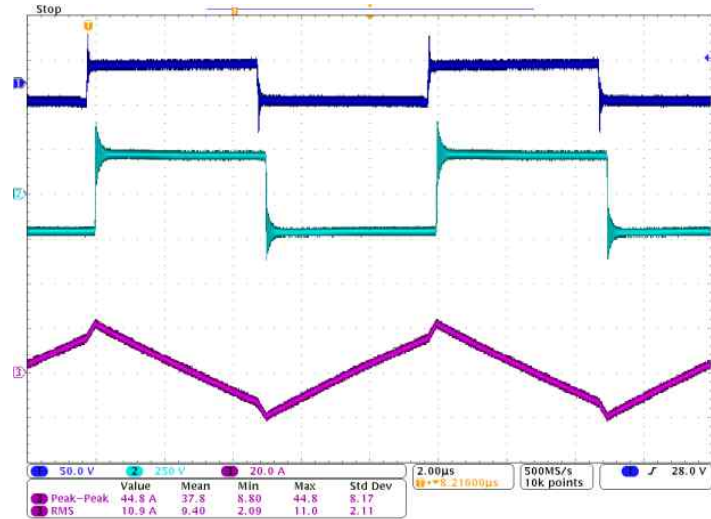

(a)

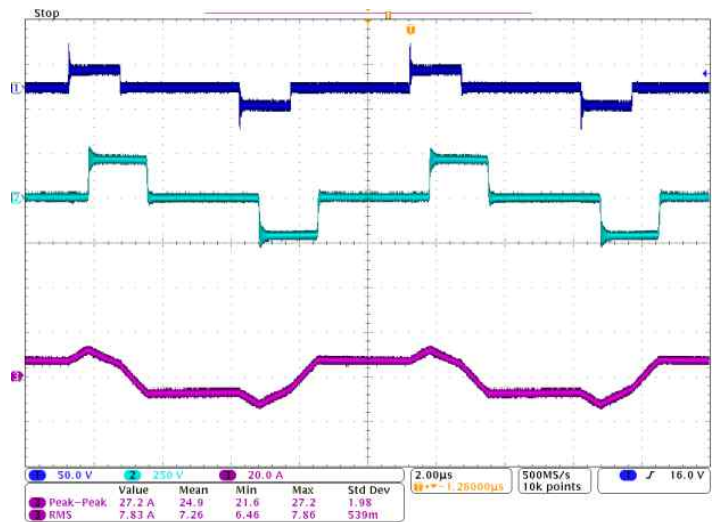

(c)

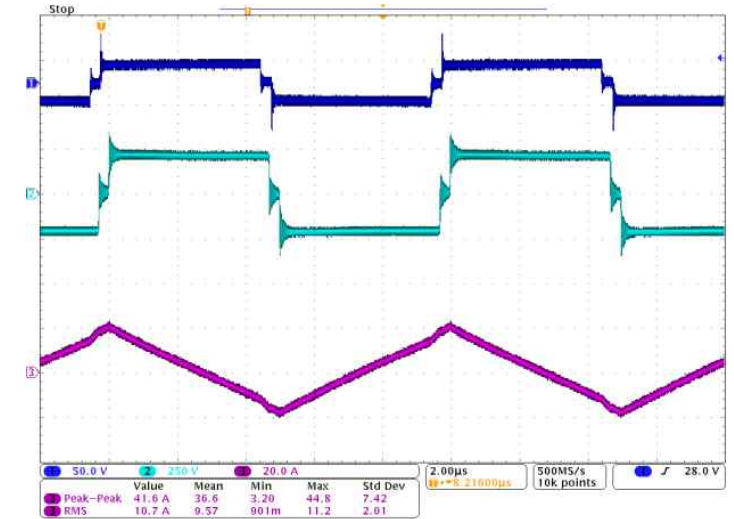

(b)

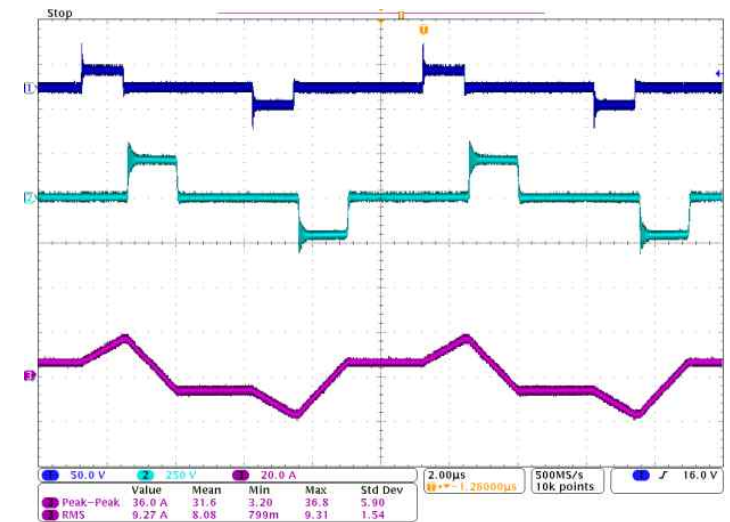

(d)

Fig. 8. Experimental waveform of $v_{T 1}, v_{T 2}$, and $i_{L}$ using the CPS and different operating modes of DPS to deliver the same output power of 50W with $d=1.8$. (a) CPS. (b) DPS_II. (c) DPS_III. (d) DPS_IV.

another degree of freedom. However, as shown in Fig. 7 (c), the rms current with DPS_I is higher than the benchmark for the whole range of $P_{o}$. In addition, the performance of the rms current with DPS_IV also deteriorates for a majority of the operating range.

2) Operating Mode of DPS: For the majority of the operating range, DPS_III shows the best performance irrespective of the optimization target. This result is also consistent with the analysis of the power transfer features, which is shown in Section 3A. DPS_IV shows the minimum rms current for a small zone of the operating range. However, both the peak current and the rms peak of DPS_IV increased sharply with the output power due to the short duration of the effective power transfer interval. For DPS_I, the performance, especially the rms current, is worse than that of the CPS control. DPS_II, always shows a slightly better performance than the CPS and it can be used for high-power applications.

3) Optimized Target: With the minimum peak current as the optimization target, the improvement with DPS is clear and the effective range is also wide, which shows the advantages of the DPS in minimizing switching loss.

4) Voltage Conversion Ratio: The effect of the DPS is clear when the voltage conversion ratio $d$ is far away from unity. The average reduction of the peak current with DPS_III is $17.6 \%$ when $d=1.5$. 


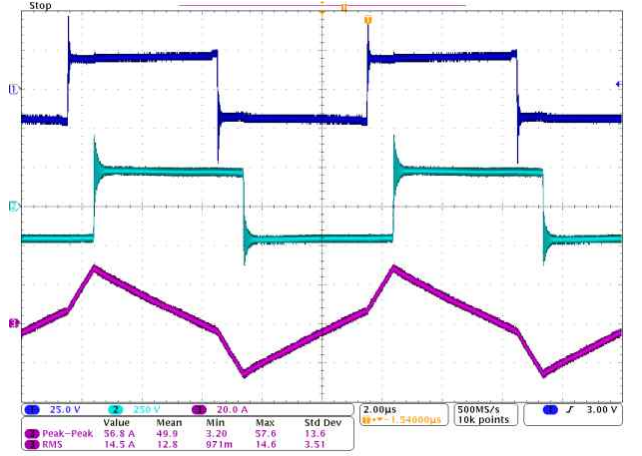

(a)

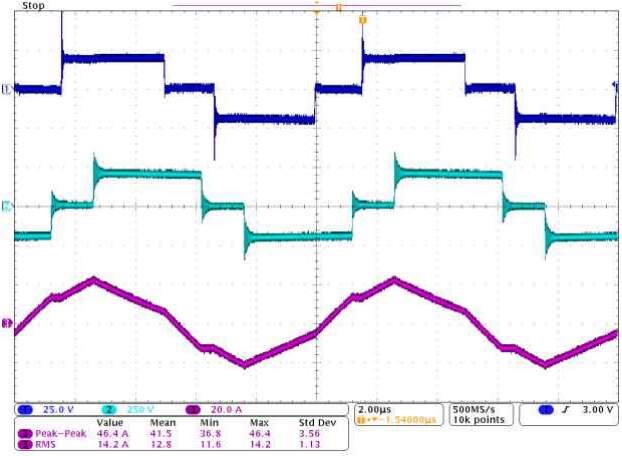

(b)

Fig. 9. Experimental waveform of $v_{T 1}, v_{T 2}$, and $i_{L}$ using the CPS and DPS_III to deliver the same output power of 200W with $d=1.8$. (a) CPS. (b) DPS III.

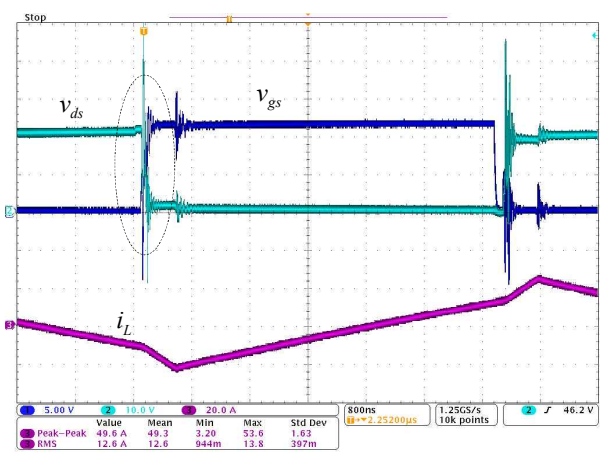

(a)

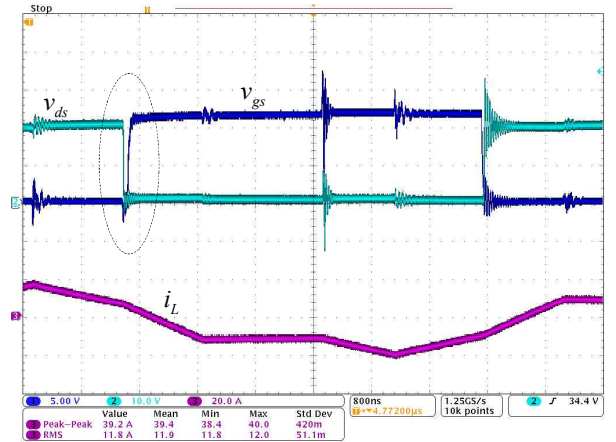

(b)

Fig. 10. Experimental waveform of ZVS condition for $Q_{13}, Q_{14}$ leg of the primary side. (a) CPS. (b) DPS_III.

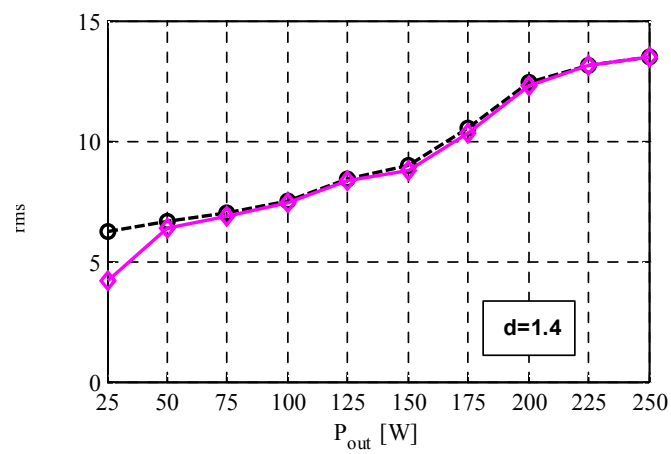

(a)

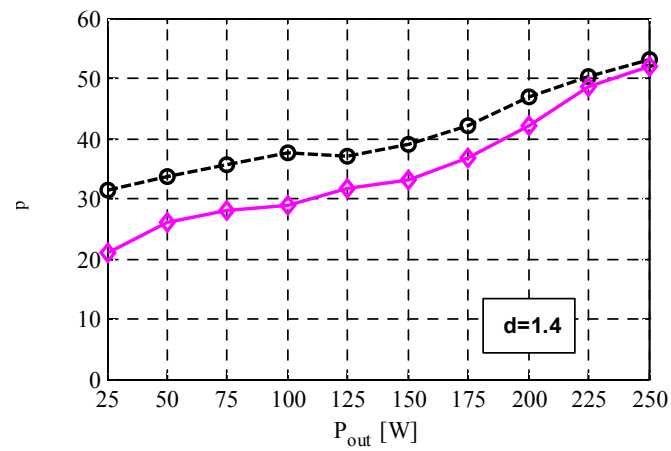

(c)

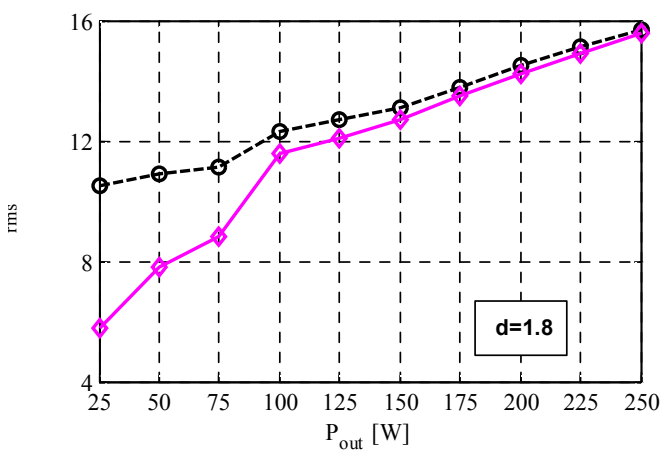

(b)

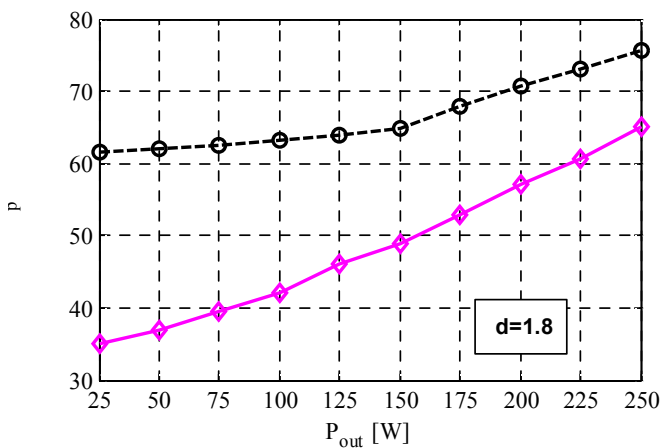

(d)

Fig. 11. Measured DAB converter rms current (top) and summation of the current at the switching angles (bottom) versus the delivered power for different voltage conversion ratio and two control strategies: CPS (dashed black line with circle marks) and DPA_III (solid magenta line with diamond marks). 


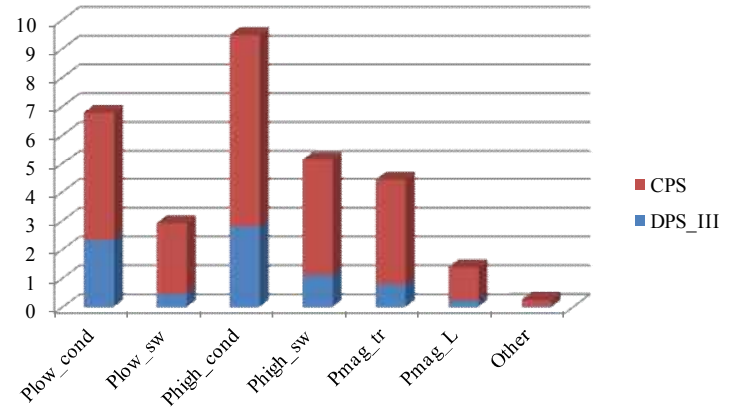

Fig. 12. Comparison of loss breakdown distribution with CPS and DPS III.

5) Output Power Range: the effect of the DPS is clear for light-load operation. The reduction of the peak current with DPS_III is $9.7 \%$ when $P_{o}=150 \mathrm{~W}$, while the corresponding reduction with the same mode is $51.9 \%$ when $P_{o}=25 \mathrm{~W}$.

\section{EXPERIMENTAL RESULTS}

A DAB converter is designed and constructed to verify the analysis and optimization with the DPS. The specifications of the prototype are summarized in Table VI. The ferrite core model is RM14-3F3, and Litz wires are used for the windings so as to minimize the losses resulting from skin and proximity effects. The digital control algorithm is implemented with a TMS320F2808 DSP. In the test, the phase-shift pair is determined by achieving the minimum inductor peak current, and a two-input table was adopted as the phase-shift pair reference. Power MOSFETs (PSMN5R5-60YS and STB13NM60N) are adopted as devices for the primary and secondary H-bridges of the DAB converter, respectively.

\section{A. Experimental Waveforms of the Operating Modes}

TABLE VII summarizes the measured results and compares the CPS with the different operating modes of the DPS, which are under the same test conditions in regards to the output power, input voltage, and load resistance. In this test, the boost operation with $d=1.8$ is tested for the DAB prototype. The peak and rms values of the inductor current are measured and shown in TABLE VII, which corresponds to the CPS and the different operating modes of the DPS. The inductor peak and rms currents with DPS_III are dramatically reduced and the efficiency is $14 \%$ higher than that of the CPS control. These results support the theoretical analysis, which is described in Section 3A. The experimental tests also indicate the importance of determining the optimal operating mode for the DPS since DPS_I shows a lower efficiency than the CPS.

Fig. 8 also shows steady-state experimental waveforms of the primary voltage $v_{T 1}$, secondary voltage $v_{T 2}$ and inductor current $i_{L}$ under the CPS and the various operating modes of the DPS for the same output power, 50W. In Fig. 8, the measured peak-peak value of $i_{L}$ using DPS_III is reduced from 44.8A to $27.2 \mathrm{~A}$, which results in lower switching losses. The rms value of $i_{L}$ is also reduced from 10.9A to $7.83 \mathrm{~A}$, which corresponds to reductions of the conduction losses and transformer losses.

Fig. 9 shows the experimental waveforms of $v_{T 1}, v_{T 2}$, and $i_{L}$ using the CPS and DPS_III to deliver the same output power of $200 \mathrm{~W}$. The measured efficiency is improved from $88.9 \%$ with the CPS to $90.2 \%$ with DPS_III.

Fig. 10 shows the ZVS condition of the primary side leg switch, $Q_{14}$, for " $P_{o}=100 \mathrm{~W}$ " and " $d=1.8$ ". It is easy to find that the ZVS condition is achieved for the $Q_{13}, Q_{14}$ leg of the primary side, which shows that the DPS will help to increase the number of legs with ZVS performance, as shown in Fig. 10 (c).

\section{B. RMS Current and Efficiency Comparison}

Fig. 11 shows the experimentally measured DAB converter rms current and the summation of the current at the switching angles, versus the delivered power, for two values of $d: 1.4$ and 1.8 . It covers a wide output power span, from $10 \%$ to $100 \%$ of the maximum output power $P_{\text {omax }}$. The performance of the CPS is used as a benchmark for the comparison. It can be observed in these figures that DPS_III has apparent advantages over the CPS especially in the cases of a higher voltage conversion ratio and a low power.

Based on the parameters of the prototype shown in Table VI, the estimated power loss distribution of the prototype with $P_{o}=50 \mathrm{~W}$ using the two control strategies of CPS and DPS_III is calculated and shown in Fig. 12. DPS_III shows distinguishable advantages in terms of the loss reductions in every aspect, including the low-voltage side MOSFET conduction $P_{\text {low_con }}$ and switching losses $P_{\text {low_sw, }}$ the high-voltage side MOSFET conduction $P_{\text {high_cond }}$ and switching losses $P_{\text {high } \_ \text {sw }}$, the losses of transformer $P_{\text {mag_t } t}$, the losses of inductor $P_{\text {mag_ } L}$ and other losses including the parasitic resistor losses. It can be found that the conduction losses make up the dominant part of the loss distribution. From the analysis and experiments, DPS_III effectively minimizes the conducting current by reducing the reactive power. As a result, the losses of $P_{\text {low_con }}, P_{\text {high_cond }}, P_{\text {mag_t } r}$, and inductor $P_{\text {mag } L}$ are reduced. The realization of one leg with ZVS soft-switching in the low-voltage side also contributes to the reduction of $P_{\text {low_sw }}$, which represents the most significant part in the power loss minimization.

Fig. 13 shows experimental efficiency curves using the CPS and DPS_III versus the output power with two different voltage conversion ratios of $d=1.4$ and $d=1.8$. Using the CPS, the efficiency drops remarkably when the delivered power is reduced for both conversion ratios, as shown in Fig. 13. This is mainly due to the increase of reactive power and circulating current using the CPS. However, the efficiency using the DPS remains at a relatively high value even for 
very low-power conditions, showing up to $15 \%$ higher than that measured with the CPS for the lowest output power.

\section{CONCLUSIONS}

The conventional phase-shift (CPS) control for the DAB suffers from low efficiency under the conditions of light loads and wide voltage conversion ratios. This results from the loss of ZVS and the induced significant reactive power when the voltage ratio deviates from unity. Based on the Dual-Phase-Shift (DPS) control, this study provides an in-depth analysis and optimization method to enhance the conversion efficiency by minimizing the reactive power and by extending the ZVS soft-switching region. The key features of the four operating modes with the DPS are characterized to determine the optimal mode for improving conversion efficiency. The mathematical expressions of the reactive power for each mode are obtained and the performance in terms of reactive power reduction is illustrated with respect to a wide output power range and voltage conversion ratio. The ZVS soft-switching boundary of the DPS is also obtained and compared with that of the CPS control. With the determined optimal operating mode, the peak and rms values of the inductor current are mathematically derived and adopted as the performance indices to find the optimal phase-shift pairs.

A prototype of a DAB converter was built to verify the theoretical analysis with the DPS. The experimental results reveal that the DPS has the capability of extending the ZVS soft-switching range and minimizing the reactive power for a wide operating range. The control implementation of the DPS demonstrates the efficiency improvement for every aspect of the load conditions and the voltage conversion ratios. In the case of low power, which is $10 \%$ of the rated capacity, a $15 \%$ efficiency improvement is observed when compared with the CPS control.

\section{ACKNOWLEDGMENT}

This research was supported by University Research Development Fund (RDF-12-2-4), Jiangsu Province University Natural Science and Research Program (13KJB470013), and the National Nature Science Foundation of China (5140070155).

\section{REFERENCES}

[1] R. W. A. A. De Doncker, D. M. Divan, and M. H. Kheraluwala, "A three-phase soft-switched high-power-density DC/DC converter for high-power applications," IEEE Trans. Ind. Appl., Vol. 27, No. 1, pp. 63-73, Jan./Feb. 1991.

[2] D.-K. Jeong, M.-H. Ryu, H.-G. Kim, and H.-J. Kim, "Optimized design of Bi-directional dual active bridge converter for low-voltage battery charger," Journal of Power Electronics, Vol. 14, No. 3, pp. 468-477, May 2014.
[3] N. Tan, T. Abe, and H. Akagi, "Design and performance of a bidirectional isolated DC-dc converter for a battery energy storage system," IEEE Trans. Power Electron., Vol. 27, No. 3, pp. 1237-1248, Mar. 2012.

[4] H. Qin and J. W. Kimball, "Solid-state transformer architecture using AC-AC dual-active-bridge converter," IEEE Trans. Ind. Electron., Vol.60, No.9, pp.3720-3730, Sep. 2013.

[5] S. P. Engel, N. Soltau, H. Stagge, and R. W. De Doncker, "Dynamic and balanced control of three-phase high-power dual-active bridge DC-DC converters in DC-grid applications," IEEE Trans. Power Electron., Vol. 28, No.4, pp. 1880-1889, Apr. 2013.

[6] F. Krismer and J. W. Kolar, "Efficiency-optimized high-current dual active bridge converter for automotive applications," IEEE Trans. Ind. Electron., Vol. 59, No. 7, pp. 2745-2760, Jul. 2012.

[7] B. Zhao, Q. Song, W. Liu, and Y. Sun, "Overview of dual-active-bridge isolated bidirectional DC-DC converter for high-frequency-link power-conversion system," IEEE Trans. Power Electron., Vol. 29, No. 8, pp. 4091-4106, Aug. 2014.

[8] G. G. Oggier, G. O. Garcia, and A. R. Oliva, "Modulation strategy to operate the dual active bridge DC-DC converter under soft switching in the whole operating range," IEEE Trans. Power Electron., Vol. 26, No. 4, pp. 1228-1236, Apr. 2011

[9] A. F. Burke, "Batteries and ultracapacitors for electric, hybrid, and fuel cell vehicles," in Proc. IEEE, Vol. 95, No. 4, pp. 806-820, 2007.

[10] J. Everts, F. Krismer, J. Van den Keybus, J. Driesen, and J. W. Kolar, "Optimal ZVS modulation of single-phase single-stage bidirectional DAB AC-DC converters," IEEE Trans. Power Electron., Vol. 29, No. 8, pp. 3954-3970, Aug. 2014.

[11] H. Wen, B. Su, and W. Xiao, "Design and performance evaluation of a bidirectional isolated dc-dc converter with extended dual-phaseshift scheme," IET Power Electron., Vol. 6, No. 5, pp. 914-924, May 2013.

[12] T. Haimin, A. Kotsopoulos, J. L. Duarte, and M. A. M. Hendrix, "Transformer-coupled multiport ZVS bidirectional DC-DC converter with wide input range," IEEE Trans. Power Electron., Vol. 23, No. 2, pp. 771-781, Mar. 2008.

[13] F. Krismer, S. Round, and J. W. Kolar, "Performance optimization of a high current dual active bridge with a wide operating voltage range," in Proc. 37th IEEE PESC, pp. 909-915, 2006.

[14] N. Schibli, "DC-DC converters for two-quadrant operation with controlled output voltage," in Proc. 9th EPE, pp. 1-9, 1999.

[15] Z. Haihua and A. M. Khambadkone, "Hybrid modulation for dual-active-bridge bidirectional converter with extended power range for ultracapacitor application," IEEE Trans. Ind. Appl., Vol. 45, No. 4, pp. 1434-1442, Jul./Aug. 2009.

[16] H. Haibing, W. Al-Hoor, N. H. Kutkut, I. Batarseh, and Z. J. Shen, "Efficiency improvement of grid-tied inverters at low input power using pulse-skipping control strategy," IEEE Trans. Power Electron., Vol. 25, No. 12, pp. 3129-3138, Dec. 2010.

[17] B. Hua and C. Mi, "Eliminate reactive power and increase system efficiency of isolated bidirectional dual-active-bridge DC-DC converters using novel dual-phase-shift control," IEEE Trans. Power Electron., 
Vol. 23, No. 6, pp. 2905-2914, Nov. 2008.

[18] B. Hua, N. Ziling, and C. C. Mi, "Experimental comparison of traditional phase-shift, dual-phase-shift, and model-based control of isolated bidirectional DC-DC converters," IEEE Trans. Power Electron., Vol. 25, No. 6, pp. 1444-1449, Jun. 2010.

[19] A. K. Jain and R. Ayyanar, "PWM control of dual active bridge: comprehensive analysis and experimental verification," IEEE Trans. Power Electron., Vol. 26, No. 4, pp. 1215-1227, Apr. 2011.

[20] F. Krismer and J. Kolar, "Closed form solution for minimum conduction loss modulation of DAB converters," IEEE Trans. Power Electron., Vol. 27, No. 1, pp. 174-188, Jan. 2012.

[21] G. Ortiz, H. Uemura, D. Bortis, J. W. Kolar, and O. Apeldoorn, "Modeling of soft-switching losses of IGBTs in high-power high-efficiency dual-active-bridge DC/DC converters," IEEE Trans. Electron. Devices, Vol. 60, No. 2, pp.587-597, Feb. 2013.

[22] Z. Shen, R. Burgos, D. Boroyevich, and F. Wang, "Soft-switching capability analysis of a dual active bridge DC-DC converter," in Proc. IEEE Electr. Ship Technol. Symp., pp. 334-339, 2009.

[23] H. Akagi, E. H. Watanabe, and M. Aredes, Instantaneous Power Theory and Applications to Power Conditioning, IEEE Press/Wiley Interscience, p. 30, 2007.

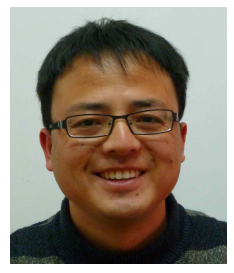

Huiqing Wen received his B.S. and M.S. degrees in Electrical Engineering from Zhejiang University, Hangzhou, China, in 2002 and 2006, respectively. He received his $\mathrm{Ph} . \mathrm{D}$. degree in Electrical Engineering from the Chinese Academy of Science, Beijing, China, in 2009. From 2009 to 2010 , he was an Electrical Engineer working with the GE (China) Research and Development Center Company, Ltd., Shanghai, China. From 2010 to 2011, he was an Engineer at the China Coal Research Institute, Beijing, China. From 2011 to 2012, he was a Postdoctoral Fellow at the Masdar Institute of Science and Technology, Abu Dhabi, United Arab Emirates. He is presently a Lecturer at Xi' an Jiaotong-Liverpool University, Suzhou, China. His current research interests include bidirectional DC-DC converters, power electronics in flexible ac transmission (FACTS) applications, electrical vehicles (EVs), and high power three-level electrical driving systems.

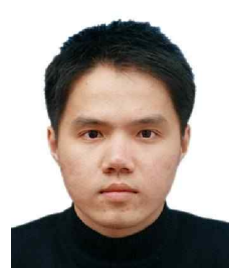

Bin Su was born in Wenzhou, China, in 1981. He received his Ph.D. degree in Electrical Engineering from Zhejiang University, Hangzhou, China, in 2010. He has authored or coauthored nine published technical papers. His current research interests include topologies, modeling and control in power electronics. 